\section{$\underset{\substack{\text { hommes } \\ \text { \& migrations }}}{ }$}

\section{Hommes \& migrations}

Revue française de référence sur les dynamiques

migratoires

$1319 \mid 2017$

Réfugiés et migrants au Liban

\title{
Territoires de la migration, territoires de la protection
}

Parcours et expériences des mineurs isolés étrangers accueillis en France

\section{Sarah Przybyl}

\section{(2) OpenEdition}

Journals

Édition électronique

URL : http://journals.openedition.org/hommesmigrations/4007

DOI : $10.4000 /$ hommesmigrations.4007

ISSN : 2262-3353

Éditeur

Musée national de l'histoire de l'immigration

Édition imprimée

Date de publication : 1 octobre 2017

Pagination : 166-170

ISBN : 978-2-919040-39-1

ISSN : 1142-852X

Référence électronique

Sarah Przybyl, «Territoires de la migration, territoires de la protection», Hommes \& migrations [En

ligne], 1319 | 2017, mis en ligne le 01 octobre 2017, consulté le 08 janvier 2021. URL : http://

journals.openedition.org/hommesmigrations/4007; DOI : https://doi.org/10.4000/

hommesmigrations. 4007 


\section{TERRITOIRES DE LA MIGRATION, TERRITOIRES DE LA PROTECTION.}

\section{PARCOURS ET EXPÉRIENCES DES MINEURS ISOLÉS ÉTRANGERS ACCUEILLIS EN FRANCE}

Par SARAH PRZYBYL, docteure en géographie, UMR Migrinter, université de Poitiers.

V isage inédit des flux migratoires contemporains, de plus en plus d'individus âgés de moins de 18 ans arrivent en Europe sans leur représentant légal. En France, ces jeunes qualifiés de « mineurs isolés étrangers " sont souvent perçus comme les victimes d'une mobilité contrainte ou comme des aventuriers ayant pris tous les risques. Enfants en danger à accueillir aujourd'hui, étrangers candidats à la régularisation de demain, le parcours atypique de prise en charge de ces mineurs révèle toutes les limites institutionnelles et politiques à l'origine de dispositifs d'accueil particuliers. Cette recherche, soutenue en décembre $2016^{1}$ au sein du laboratoire Migrinter de l'université de Poitiers, propose de se défaire de ces représentations pour mieux refléter la pluralité des situations sociales d'origine, et de décrypter les enjeux institutionnels de la protection des mineurs isolés étrangers. La présente contribution revient sur trois principaux résultats d'une investigation menée entre 2012 et 2016.

\section{La capacité ambivalente des mineurs à être les acteurs de leur parcours}

Dans le sens commun, il est admis qu'au regard de leur âge, les mineurs ne seraient pas capables d'agir ou de penser pour eux-mêmes. De ces représentations découlent une vision où la migration des mineurs serait seulement le résultat de la volonté des adultes qui les entourent. Dans ce contexte, les mineurs sont régulièrement relégués au second plan de cette migration dont ils sont pourtant les protagonistes. En retraçant différents types de parcours migratoires et de protection, j'ai mis cette perspective à l'épreuve du terrain. À partir des témoignages que j'ai récoltés sur plusieurs lieux d'enquête, j'ai identifié différents types de migration pour rendre compte de la diversité des parcours de mobilité. La migration directe, la migration par relais ou encore celle de la débrouille

1. Ma thèse a été évaluée par un jury composé de Serge Weber, professeur de géographie à l'université Paris-Est Marne-la-Vallée (rapporteur), Gilles Séraphin, directeur de l'ONPE (rapporteur), Constance de Gourcy, maître de conférences en sociologie à l'université Aix-Marseille, Angelina Etiemble, maître de conférences en sociologie à l'université du Maine, William Berthomière, directeur de recherche au CNRS (directeur de thèse) et Daniel Senovilla-Hernández, ingénieur de recherche (directeur de thèse) de l'université de Poitiers. Voir Sarah Przybyl, "Territoires de la migration, territoires de la protection. Parcours et expériences des mineurs isolés étrangers accueillis en France ", sous la direction de William Berthomière et Daniel Senovilla Hernández, thèse de géographie, université de Poitiers, 2016. 


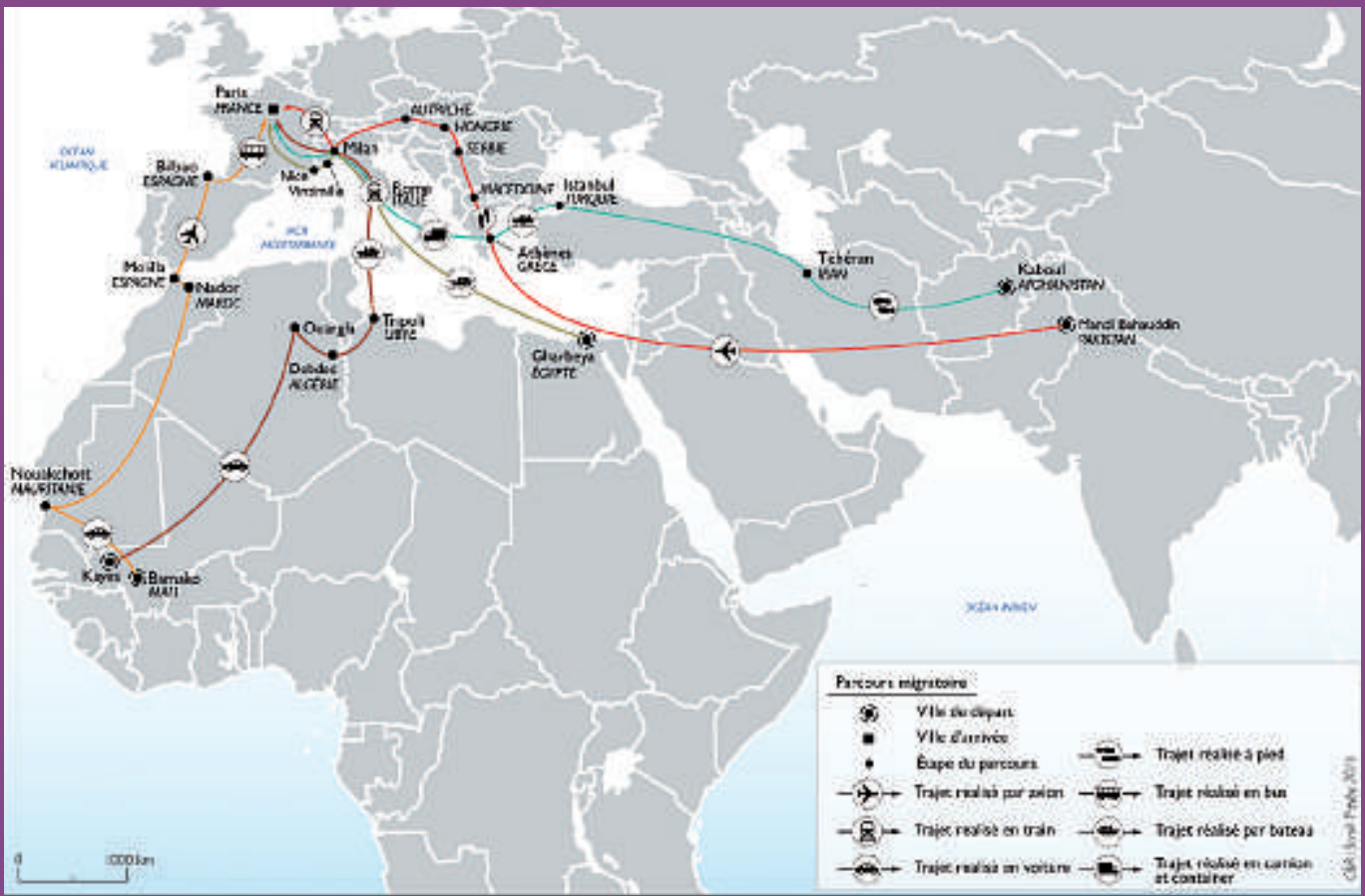

/// Carte 1. La migration par relais.

ont mis au jour différentes routes mais aussi des expériences plurielles du voyage.

À l'issue de ma recherche, deux postures ont été révélées par les expériences des jeunes enquêtés. Tout d'abord, au gré de ces parcours migratoires, les mineurs apparaissent, dans une certaine mesure, subordonnés à des figures adultes. Les différentes routes empruntées montrent qu'ils ont été obligés d'être dépendants des adultes, ou plutôt de ceux qui savaient comment passer en Europe ou assurer leur protection le temps du voyage. Cette posture de subordination n'est pas propre au temps de la migration puisqu'elle se retrouve également en France, dans les parcours de prise en charge des mineurs en danger. Dans les structures d'accueil enquêtées, les jeunes sont tributaires d'un monde où les décisions les plus importantes sont gérées par des adultes incarnés par les travailleurs sociaux, les juges pour enfants ou encore les responsables de l'Aide sociale à l'enfance (ASE). L'exemple de la prise en charge illustre la manière dont les jeunes ne disposent que d'une très faible marge de manœuvre au regard des injonctions administratives et des impératifs quotidiens auxquels ils doivent répondre pour réussir. Au cours de ma recherche, cette incapacité a été rapidement nuancée et remise en perspective. Dans les lieux de leur prise en charge, dans leur quotidien au pays d'origine, ou encore au moment de traverser la Méditerranée, les jeunes enquêtés se présentent aussi comme des acteurs des environnements où ils se trouvent. L'exemple de Tanger ${ }^{2}$ ou encore les monographies conduites au sein des foyers soulignent la façon dont les mineurs investissent des relations sociales et s'approprient les lieux de leur protection ou de leur migration. En m'intéressant au quotidien banal qu'ils vivent dans les espaces du départ et de l'accueil, ma recherche souligne comment les mineurs se saisissent des contraintes et 


\section{REPÉRAGE}

des opportunités pour progresser dans leur parcours de vie et les différents projets qu'ils doivent réussir. Plus que des fins connaisseurs des enjeux d'un cadre de l'accueil qu'ils maîtriseraient totalement, les mineurs révèlent leur capacité d'action dans les logiques d'appropriation des lieux et des espaces, dans un ensemble de relations sociales pris dans des enjeux institutionnels ou plus quotidiens.

\section{Les dispositifs d'accueil \\ à l'échelle française : la fabrique d'un territoire de la protection}

Après avoir dévoilé cette capacité ambivalente des mineurs à être acteurs de leur parcours, ma recherche s'est concentrée sur les logiques de construction des territoires de la protection. Pour décrypter ces dynamiques, je me suis positionnée dans le sillage des approches de géographie humaine et sociale qui considèrent le territoire comme multidimensionnel3. J'ai envisagé le territoire dans sa dimension matérielle, mais aussi plus idéelle et sensible qu'elle revêt pour les individus. Cette approche par le territoire a mis au jour plusieurs échelles de la protection. J'ai ainsi pu aborder l'agencement territorial d'une politique de la protection mais aussi comprendre les logiques individuelles d'appropriation des acteurs sur le terrain.

Du point de vue des lieux de leur arrivée, la situation des mineurs isolés étrangers a permis de proposer une lecture des territoires de la protection depuis les dynamiques politiques et institutionnelles qui structurent les réalités de l'accueil. Ma thèse dessine les contours du territoire tel qu'il est construit par le politique. Aujourd'hui, dans l'héritage de la décentralisation des compétences de l'État aux départements, la protection des mineurs isolés étrangers relève exclusivement des conseils départementaux. Au fil des années, l'inégale répartition de l'arrivée des mineurs a créé des situations de saturation de certains dispositifs d'accueil.

Dans ce contexte, pour soulager certains départements, les négociations entre l'État et ces collectivités ont abouti à une circulaire datant de mai 2013 organisant la réorientation des jeunes dans d'autres départements. Jusqu'alors circonscrits à quelques localités, ces débats ont conduit à ce que l'ensemble du territoire soit concerné par les mineurs isolés étrangers. Dans 
cette volonté de rétablir l'équilibre, des enjeux politiques et institutionnels ont émergé pour mieux souligner toute la tension qui existe entre les différents départements français, et comment les jeunes sont devenus un fardeau et une variable d'ajustement pour les autorités. Dans ces débats en faveur d'une nouvelle répartition territoriale, les jeunes sont apparus comme des individus que l'on peut déplacer à souhait, comme si ces derniers n'avaient aucune attache. Au détriment parfois de l'intérêt supérieur de l'enfant, l'organisation de la présence des jeunes a permis de mieux satisfaire les préoccupations de certains élus départementaux.

Cette approche du territoire par les politiques a été complétée par une approche micro-géographique. Plus proche des jeunes et des acteurs, j’ai montré que la protection n'est pas seulement une affaire de politique ou d'institution, mais aussi et surtout d'acteurs individuels qui agissent au quotidien.J'ai alors adopté une posture empirique radicale ancrée dans les réalités du terrain pour rendre compte des dynamiques à l'œuvre dans un même lieu et porter attention à ce qui fait la vie des individus chaque jour. J'ai abordé la façon dont les mineurs mais aussi les professionnels construisent leur territoire dans les espaces du quotidien dédiés à la protection, c'est-à-dire les foyers ou les associations. À travers les différentes relations sociales, les représentations et les usages des lieux, j'ai montré comment des micro-territoires sont façonnés chaque jour par des individus qui jonglent avec des impératifs quotidiens, mais également à travers le jeu de distance et de proximité qui s'instaure avec l'environnement immédiat.

Dans les foyers de l'enfance où ils vivent un quotidien institutionnel marqué par la promiscuité, les mineurs mais aussi les professionnels se regroupent dans des lieux qui ont un sens pour eux. Ces espaces de retrouvailles comme les chambres, les pas de porte ou encore les salles de jeu sont un moyen pour les individus de se définir dans un cadre institutionnel particulier. Lieux et individus se définissent réciproquement pour former des micro-territoires de la protection. Ma recherche montre que les territoires de la protection ne se limitent pas seulement aux frontières étatiques et départementales mais se fabriquent aussi par les expériences des individus.

\section{Les défis méthodologiques d'une recherche auprès d'un public vulnérable}

Un des résultats de ma thèse souligne enfin un enjeu de taille dans les recherches menées avec ces mineurs et engage une réflexion méthodologique. Les mineurs représentent une population dont l'âge et les parcours de vie nécessitent la mise en place de méthodes d'enquête flexibles qui doivent s'adapter aux réalités du terrain. Au fil de leur prise en charge, les mineurs sont tellement sollicités que leur récit, leur parole, leur subjectivité ne sont plus des moyens de s'exprimer mais Ma recherche montre que les territoires de la protection ne se limitent pas seulement aux frontières étatiques et départementales mais se fabriquent aussi par les expériences des individus. deviennent un objet de contrôle de leur minorité et de la véracité des informations qu'ils abordent. La parole n'est plus un outil pour communiquer mais constitue un outil de vérification. Ce constat m'a conduit à montrer que plus que la méthode en elle-même, c'est la négociation de l'enquête qui détermine grandement le déroulement des échanges avec les jeunes.

Enquêter auprès de ces mineurs nécessite une relation de confiance que seul permet le temps long. Le temps est donc une donnée centrale de mon approche. Il y a, d'une part, le temps long des périodes d'enquête et des immersions sur le terrain où j'ai vécu aux côtés des enquêtés. D’autre part, il y a le temps long du suivi pour aborder le devenir des mineurs enquêtés au fil de ma thèse. Ce 


\section{REPÉRAGE}

parti-pris m'a permis de recueillir les histoires de jeunes ayant quitté le dispositif d'accueil pour mieux comprendre leurs expériences à l'âge de la majorité et recueillir une parole plus libérée et plus maîtrisée. Parier sur le long terme m'a permis de rester en accord avec mon souhait de ne pas forcer l'élaboration des relations. Cette posture passive en apparence suppose d'admettre que la relation puisse ne pas se faire. Elle implique de laisser certains éléments à la variable de l'imprévu et permet de respecter l'intégrité des jeunes. La recherche avec des mineurs suppose d'accepter de recevoir un récit brut, fait d'incohérences, et parfois même de laisser le cours de l'échange être totalement contrôlé par les mineurs. L'issue méthodologique que j'ai proposée réside dans une approche qui veut que l'enquêteur s'adapte à l'enquêté. J'ai ainsi affirmé avec ma recherche que les ambitions scientifiques doivent s'accommoder de la parole livrée par les mineurs. Comme une référence à mes premiers résultats, je soutiens avec ce point que les jeunes sont tout à fait capables de parler d'eux mais que l'obstacle réside dans les attentes de l'enquêteur à l'égard d'un discours attendu, rêvé ou de l'aveu que seul le scientifique pourrait obtenir.

\section{Terrains rtalisés en France}

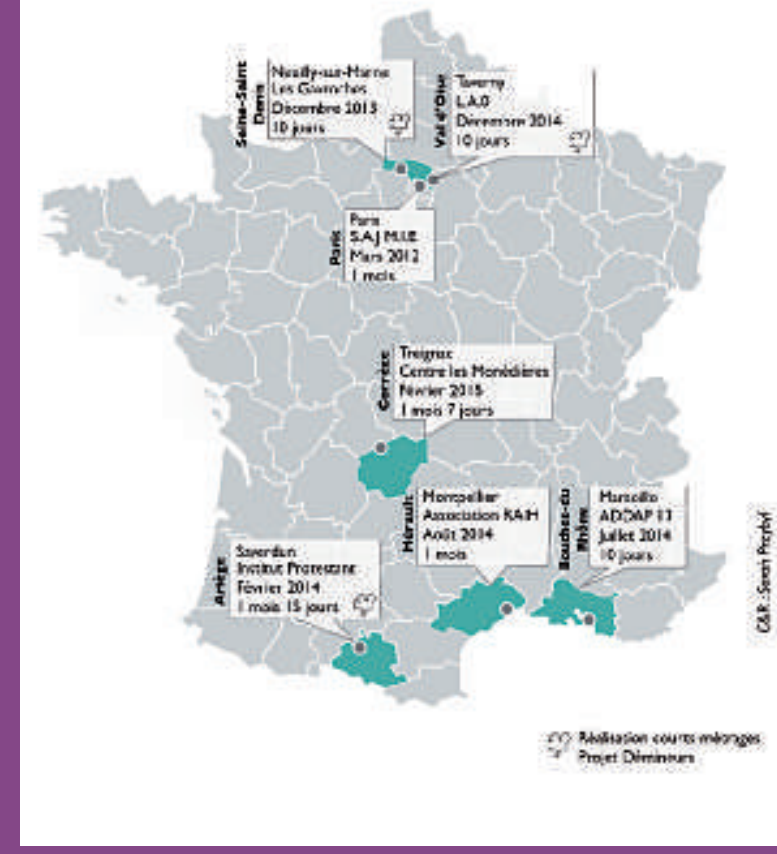

\section{/// Carte 3. Terrain d'enquête.}

Ce cadrage théorique a été soutenu par un dispositif méthodologique tenant compte à chaque instant de la singularité des mineurs enquêtés. Aussi, ma thèse propose une réflexion sur les enquêtes qualitatives mobilisables avec ce public. À travers le projet Démineurs (www.demineurs.com), elle retrace l'intérêt de l'usage d'outils alternatifs comme l'audiovisuel et interroge le sens de la relation d'enquête et de l'engagement dans le cadre de la réalisation d'entretiens semi-directifs.). 